\title{
Essai d'approche positive des nouveaux risques sociaux
}

Towards a positive approach on new social risks

\section{Catherine Pollak}

\section{(2) OpenEdition}

\section{Journals}

Édition électronique

URL : http://journals.openedition.org/travailemploi/4952

DOI : $10.4000 /$ travailemploi.4952

ISSN : 1775-416X

Éditeur

DARES - Ministère du Travail

Édition imprimée

Date de publication : 15 mars 2011

Pagination : 67-77

ISSN : 0224-4365

Référence électronique

Catherine Pollak, "Essai d'approche positive des nouveaux risques sociaux», Travail et Emploi [En ligne], 125 | janvier-mars 2011, mis en ligne le 12 janvier 2012, consulté le 19 avril 2019. URL : http:// journals.openedition.org/travailemploi/4952 ; DOI : 10.4000/travailemploi.4952 


\title{
Essai d'approche positive des nouveaux risques sociaux
}

\author{
Catherine Pollak $\left.{ }^{*}\right)$
}

\begin{abstract}
Cet article vise à clarifier les débats normatifs de la littérature sur la réforme des États Providence en proposant une approche positive des "nouveaux risques sociaux». L'hétérogénéité des risques considérés dans cette littérature pose la question du champ et de la spécificité de la protection sociale. Une définition substantielle permettant de délimiter objectivement le champ des "nouveaux risques sociaux» apparaît donc nécessaire mais aussi possible. Il s'agit certes de reconnaître que le risque social est une construction, et qu'en tant que tel il ne peut être identifié a priori. Certains risques semblent toutefois avoir un potentiel particulier à être sociaux. Pour en délimiter le champ, l'auteure propose une grille d'identification à partir de quatre critères : l'indépendance entre le risque et la responsabilité, les conséquences économiques de la réalisation du risque, leur caractère assurable, et leur répartition socialement inégale.
\end{abstract}

Cet article se propose, à partir d'une réflexion sur la récurrence actuelle de la référence à de «nouveaux risques sociaux» dans les discours de réforme des États Providence, de proposer une définition substantielle de ces risques. L'hétérogénéité des définitions considérées au sein de la littérature proposant une réforme des États Providence pose la question du champ des risques sociaux et de la politique sociale. En élargissant ce champ, ne risque-t-on pas d'entretenir une demande infinie de protection sociale? À l'inverse, en proposant de gérer les risques sociaux au même titre et par les mêmes instruments que d'autres risques économiques, ne risque-t-on pas de retirer à la politique sociale toute sa spécificité?

Afin de clarifier ces débats, nous défendons ici l'idée qu'une définition objective des risques sociaux est nécessaire et possible. Notre argumentation suivra les étapes suivantes : nous montrons que l'analyse conceptuelle de la littérature des «nouveaux risques sociaux» révèle que leurs frontières sont floues (1), ce qui accentue les paradoxes du recours à cette notion pour penser la réforme de la protection sociale (2). Disposer d'une définition substantielle des nouveaux risques sociaux permettrait de considérer la question sous un nouvel angle : ainsi, tout en reconnaissant que le caractère social $\mathrm{du}$ risque est le fruit d'une construction sociale (3), il est possible d'identifier certaines propriétés objectives des risques sociaux (4), qui permettent de délimiter le champ des nouveaux risques ayant le potentiel d'être sociaux (5).

(*) Centre d'économie de la Sorbonne; catherine.pollak@univparis1.fr

\section{Les frontières floues des «nouveaux risques sociaux"}

Le recours au terme de "risques sociaux» pour caractériser l'organisation de la protection sociale est aujourd'hui incontournable. Dans sa genèse de l'État Providence, François Ewald (1986) a montré que cette notion est à l'origine de la construction de l'État Providence en France et a largement contribué à légitimer son action. Le droit de la Sécurité sociale y fait explicitement référence pour définir son objet et les définit comme les «risques de toute nature susceptibles de réduire ou de supprimer la capacité de gain [des travailleurs et de leur famille]» (Art. L. 111-1). Traditionnellement, ces situations prises en charge par la Sécurité sociale sont regroupées en cinq domaines : la maladie, la vieillesse, la famille et le logement, le chômage, et la pauvreté et l'exclusion. On a vu se développer depuis une dizaine d'années des travaux posant la question de l'adaptation de l'architecture de l'État Providence aux nouveaux besoins des individus. Cette littérature propose de reconsidérer la prise en charge des risques sociaux, soit en insistant sur le développement de "nouveaux risques sociaux» dont la couverture est insuffisante, soit en promouvant une reconfiguration de la protection sociale dans une stratégie de "gestion des risques sociaux» étendue à ces nouveaux risques.

\section{Les "nouveaux risques sociaux" ou la crise de la protection par les États Providence}

La référence à la notion de «nouveaux risques sociaux » est devenue courante dans de nombreux travaux européens de science politique portant sur 
l'état actuel des États Providence (par exemple ESPING-ANDERSEN et al., 2001; TAYLOR-GOOBY, 2004). À travers l'utilisation de ce terme, il s'agit de pointer l'inadaptation de la protection sociale face à l'émergence de situations nouvelles et d'insister sur la nécessité de réformer ces États Providence. En témoigne la définition souvent reprise de Giuliano Bonoli $(2006$, p. 5) : «les nouveaux risques sociaux sont perçus comme les situations dans lesquelles les individus perçoivent des pertes de bien-être et qui résultent des transformations socio-économiques qui ont accompagné l'émergence des sociétés postindustrielles » (1). Dans cette littérature, le terme est utilisé pour mettre en avant l'existence de nouveaux besoins insuffisamment ou non pris en charge par la protection sociale, ces risques étant considérés comme nouveaux par leur nature (par exemple le SIDA) ou par leur ampleur (chômage de longue durée, la pauvreté, les problèmes liés à l'immigration, la monoparentalité) (PALIER, 2002). Il permet de répondre aux arguments financiers en rappelant le rôle politique, économique et social de la protection sociale. L'idée est que la crise de l'État Providence n'est pas qu'une crise de financement mais aussi une crise de couverture. Elle résulte d'une protection insuffisante de nouvelles situations ou d'un agencement inadapté de la prise en charge qui a pu conduire à son tour à un accroissement des inégalités (Palier, 2002; Ferrera, Rhodes, 2000).

L'acception de la notion de "nouveaux risques sociaux » est vaste et hétérogène. Parmi les risques les plus souvent évoqués, on peut citer : le care et la conciliation entre vie familiale et vie professionnelle, l'instabilité des structures familiales et le risque de pauvreté des familles monoparentales et des enfants; le risque de chômage de longue durée, de pauvreté laborieuse, d'insuffisance ou d'obsolescence des compétences, de déclassement, de baisse des capacités de gain, d'éviction précoce et de marginalisation du marché du travail, d'absence ou de perte de droits, etc.

Dans cette littérature, l'identification des risques sociaux passe principalement par la désignation de groupes vulnérabilisés. Ainsi, les «nouveaux risques sociaux 》 affectent des groupes qui étaient autrefois préservés ou moins nombreux (les familles monoparentales, les personnes âgées dépendantes, les jeunes, les migrants, les personnes exclues du droit commun de la protection sociale), même si les avis divergent sur les populations qui seraient les plus touchées. De fait, les frontières de ces «nouveaux risques sociaux» sont floues, puisqu'ils peuvent désigner des situations pour lesquelles il n'existe pas de prise en charge organisée, mais aussi des risques déjà partiellement couverts qui touchent de nouvelles populations. Ils peuvent enfin désigner des risques nouvellement reconnus, déjà couverts effectivement par des prestations éparses et lacunaires, qui se sont consolidées peu à peu jusqu'à donner lieu à une reconnaissance administrative.

\section{La "gestion des risques sociaux" comme nouvelle stratégie de gestion des États Providence}

Un second pan de la littérature, plutôt porté par des économistes, mobilise le recours aux nouveaux risques sociaux pour plaider en faveur d'une refondation du système de prise en charge selon l'idée de «gestion des risques sociaux». Il s'agit de poser les principes d'un système de protection sociale élargi qui reposerait sur la combinaison de différentes stratégies de gestion du risque (prévention, atténuation, réaction), afin d'assurer une sécurité économique aux individus tout en encourageant la «prise de risque». Il en existe deux versions principales.

La plus connue est celle de la Banque Mondiale (Holzmann, JoRgensen, 2001), dont l'approche de la protection sociale est universalisante et gestionnaire. Elle préconise une vision large des mécanismes de gestion du risque agissant en amont et en aval. Elle part de l'analyse des acteurs de la gestion du risque qui repose sur trois institutions protectrices, à savoir le marché, la famille et les autorités publiques. Le risque social est perçu comme celui de ne plus pouvoir se reposer sur les deux premières, ce qui conduit à préconiser une intervention de l'État en dernier ressort. Le cœur de la stratégie est de combiner un filet de sécurité minimal, permettant d'assurer la satisfaction des besoins primaires, à des incitations à la prise de risque pour accroître le revenu des individus.

Une version alternative de la gestion des risques sociaux est proposée par Günther Schmid (2006). S'inspirant de l'approche des marchés transitionnels du travail, cette version met l'accent sur les risques associés aux transitions entre statuts d'emploi et leurs conséquences sur la sécurité des revenus et la qualité de l'emploi. Elle reprend le principe de la combinaison de différentes stratégies de gestion des risques en considérant ce concept comme «une opportunité morale pour reconsidérer l'équilibre entre la solidarité et la responsabilité individuelle» (2) (p. 1). Elle se distingue de la première vision au niveau de la place accordée à la prise en charge collective (l'intervention collective et les partenaires sociaux doivent y occuper une place centrale), le champ d'intervention (les mesures de redistribution font partie intégrante de la gestion des risques sociaux), et les moyens d'encourager la prise de risque (droits individualisables adaptés aux nouvelles formes de carrières). 
Dans les deux cas, la promotion d'une stratégie de «gestion du risque social» dépasse le cadre traditionnel de la protection sociale - à savoir la compensation des risques - puisqu'ils sont appelés à être gérés également en amont par des politiques de prévention. Cette stratégie se veut globale et repose sur une vision très élargie des risques sociaux : a priori, tous les risques conduisant à des pertes de bien-être peuvent y être rattachés. Il ne s'agit pas d'énumérer les risques sociaux dont la prise en charge serait prioritaire ou d'en délimiter les contours à partir de leurs caractéristiques. Le cœur de la gestion des risques sociaux repose sur une nouvelle manière de considérer les responsabilités individuelles et collectives pour chaque type de risque : cette littérature se focalise sur les causes (internes ou externes) des risques et les acteurs intervenant dans leur gestion, et cherche à poser les principes d'une gestion optimale de tous types de risques.

\section{Les paradoxes du recours aux "nouveaux risques sociaux" pour penser la réforme de la protection sociale}

Cette littérature récente sur les «nouveaux risques sociaux » ravive les controverses idéologiques sur la place des stratégies de gestion du risque dans les réformes de la politique sociale. On peut dans une certaine mesure attribuer ces controverses aux implications sémantiques de la notion même de risque, dont l'utilisation dans le domaine de la protection sociale implique une légitimation du caractère principalement assurantiel de la prise en charge, voire d'une individualisation des responsabilités.

\section{L'insuffisance de la notion de risque pour penser l'État social}

La centralité du recours aux risques sociaux suscite des réserves portant sur la place qui lui est accordée pour penser le système de protection sociale dans sa spécificité et dans sa globalité.

Le caractère probabilisable du risque (qui le distingue par exemple du danger) renvoie au paradigme assurantiel de la protection sociale, qui certes légitime largement l'action de l'État, mais qui ne rend pas compte de la spécificité des objectifs et des techniques du système de protection sociale (neutralité actuarielle vs redistribution, assurance et compensation $v s$ assistance et services gratuits, assurance $v s$ prévoyance et réallocation des revenus sur le cycle de vie, etc.) (Blanchet, 1996; Ramaux, 2007). D'ailleurs, des propositions telles que l'allocation universelle attestent de la possibilité de penser un système de protection sociale sans référence au risque.
De plus, certains auteurs contestent la centralité du recours aux risques sociaux du fait de leur insuffisance pour penser l'État social dans sa globalité. Son action, même en ce qui concerne le domaine de la protection sociale, ne se limite pas à la stricte compensation des conséquences néfastes des risques sociaux, mais recouvre des objectifs d'amélioration qualitative visant à garantir un certain bien-être social, voire un mieux-être social (RAmaux, 2007). Pour certains, les connotations négatives associées au terme de risque rendent son utilisation obsolète à une époque où les droits couvrent des situations qui ne sont plus autant synonymes de détresse pour les travailleurs (Concialdi, 1999). Ces travaux visent aussi à rappeler que l'action de l'État social dépasse la couverture des risques sociaux. Dans la mesure où ils cherchent à penser l'État social, et même la protection sociale, hors du registre assurantiel, ils se distinguent de la vision englobante de la «gestion des risques sociaux», qui inclut (dans la gestion des risques) les mesures de politique générale visant à réduire la vulnérabilité et l'instabilité des revenus.

\section{L'État social réformé : un "État minimal" post-assurantiel?}

L'omniprésence de la notion de risque dans les discours de réforme de l'État social ne peut également être dissociée de courants de pensée qui, dès les années 1970, avaient fait du risque un objet de réflexion majeur des sociétés post-industrielles. Le recours au registre du risque ravive ainsi des controverses anciennes sur la place des techniques de gestion du risque dans les sociétés modernes et sur l'individualisation des responsabilités.

Ces controverses trouvent leur origine dans l'opposition de la sociologie de la "société du risque» à la tradition foucaldienne. Dans la première tradition, portée notamment par Ulrich BECK (1986) et Anthony GidDENs (1998), le passage à une société postindustrielle est caractérisé par l'accroissement des incertitudes et la prolifération des risques, et notamment des risques «manufacturés» (ou internes) résultant de l'intervention humaine. Ainsi, la crise de l'État Providence y est perçue comme une crise de gestion du risque social, qui était conçue pour assurer les risques externes (imputables à la nature) et ne s'est pas adaptée à l'accroissement des risques manufacturés. Cette conceptualisation de la «société du risque», à l'origine de la "Troisième Voie» de Tony Blair, a aussi contribué à inspirer les promoteurs de la «gestion des risques sociaux».

On peut relever trois points de critique majeurs qui lui ont été opposés et qui permettent de comprendre une partie des enjeux du débat autour de la "gestion des risques sociaux». Le premier porte sur l'accroissement des dispositifs visant à gérer les risques, ce qui, dans la lignée de Michel Foucault (1977) constitue un instrument de domination et d'exercice du pouvoir entretenant une demande infinie de 
sécurité ( $c f$. aussi CASTEL, 2003). Le deuxième porte sur l'importance qui est accordée à la répartition des risques dans la structure sociale, qui conduit à reconsidérer les principales inégalités comme des inégalités face au risque et à évincer le rôle majeur des structures traditionnelles (TAYLOR-GOOBY, ZINN, 2006). Enfin, le glissement sémantique de la notion de risque (des risques externes aux risques internes), associé à une conception nouvelle des inégalités, correspond à un glissement des responsabilités collectives aux responsabilités individuelles. Ainsi, la focalisation sur les techniques de gestion des risques peut occulter la spécificité et la dimension sociale des problèmes rencontrés et justifier une individualisation de leur prise en charge.

Toutefois, malgré les critiques contestant la centralité du risque pour penser la réforme de la protection sociale, la référence à cette notion n'a pas été réellement concurrencée par d'autres dans le champ des travaux portant sur la construction et la réforme de la protection sociale (on pourrait pourtant lui opposer celles de besoin, de bien-être, de droits, ou encore de sécurité). La lecture en termes de risques sociaux présente des atouts forts qui peuvent expliquer cette pérennité : étant à l'origine de la construction du droit social et au cœur des mécanismes assurantiels, elle permet de légitimer l'intervention collective; englobante et évolutive, elle permet de penser les réformes; enfin, parce qu'elle considère à la fois les situations de détresse comme socialement inacceptables et les prises de risque comme constructives, et qu'elle ne préjuge pas de la couverture nécessaire, elle est compatible avec des conceptions très différentes de la prise en charge.

Si la centralité de la notion de risques sociaux pour penser la réforme de la protection sociale est parfois contestée, ce n'est pas le recours au registre du risque qui rend la littérature sur les «nouveaux risques sociaux» inévitablement normative, mais l'absence de critères de définition objectifs de ces risques. En effet, elle se focalise sur la prise en charge souhaitable des risques, sans que leurs contours ne soient clairement identifiés, ce qui peut paradoxalement conduire à considérer comme «nouveaux risques sociaux» soit tous les risques, soit aucun. Afin d'éclairer ces débats, une clarification des contours des «nouveaux risques sociaux» semble donc nécessaire.

\section{L'impossible approche positive des risques sociaux?}

La définition tautologique habituelle, consistant à appeler risques sociaux les risques effectivement couverts par la protection sociale, atteint clairement ses limites lorsqu'il s'agit de reconnaître de «nouveaux risques sociaux». Ceci invite à se poser la question de la possibilité de développer une approche positive du risque social.

\section{Le risque social est une construction sociale}

La notion de risque social est une notion d'abord juridique qui a permis de distinguer le dommage fautif du dommage social en terme de responsabilité. Ewald (1986) date la naissance de la notion de risque social en France avec la loi du 9 avril 1898 qui établit la notion de risque professionnel pour les accidents du travail. Son analyse met l'accent sur le fait que la mutation fondamentale est juridique, car la reconnaissance des accidents du travail traduit une révolution dans la conception de la responsabilité. Elle enterre le principe de réparation basé sur la reconnaissance de la faute et de la responsabilité individuelle au profit d'une responsabilité globale et «sociale». La réparation du dommage n'incombe plus à une personne physique ou morale reconnue comme fautive. Ce passage de la faute à l'accident et au risque est révélateur de profonds changements sociaux et juridiques, qui se situent à l'origine de l'assurance sociale. Les risques sociaux sont aujourd'hui compris par les juristes comme «les événements aléatoires qui affectent la vie économique des individus en suscitant une diminution de leurs revenus ou en accroissant leurs dépenses, et qui constituent un désordre social auquel il convient de répondre» (KeSSLER, 2000, p. 11). Le Code de la Sécurité sociale énumère les différents risques reconnus et couverts par des droits sociaux. Le risque social est donc celui qui a fait l'objet d'une intervention des pouvoirs publics et qui a été caractérisé comme tel. Ainsi, «par une tautologie nécessaire, est «risque social» ce qui est couvert par la sécurité sociale» (EwALD, 1986, p. 401). Ouverte et évolutive, cette conception permet d'inclure de nouvelles garanties en fonction des nouveaux domaines d'intervention qui se créent au cours du temps (par exemple la naissance de l'assurance chômage en 1958 en France). En effet, «avec cette caractéristique que rien n'étant en soi un risque, tout peut en être un» (EwALD, 1986, p. 19).

Dans une perspective socio-historique, le risque social est conçu clairement comme une construction sociale indissociable du passage à l'assurance sociale (Rosanvallon, 1995). De façon générale, la conception sociologique du risque se focalise sur sa représentation et ses systèmes de gestion. L'efficacité de la notion tient au fait qu'elle ne désigne pas une catégorie d'événements particuliers, mais une façon de se représenter les événements, de les objectiver, étendue sans cesse à de nouveaux objets (PereTtIWatel, 2000). Le développement des études psychologiques et sociologiques a permis d'améliorer la compréhension des facteurs de perception des risques par les individus (facteurs culturels, institutionnels, technologiques, émotionnels, etc.) (Peretti-Watel, 2000; Taylor-Gooby, Zinn, 2006) 
qui évoluent avec le temps et le contexte. De même, l'avancée des techniques est fondamentale pour que des dangers puissent être perçus comme des risques. En effet, le caractère assurable du risque en est l'élément essentiel et permet de distinguer les événements qui sont du domaine du risque de ceux qui sont du domaine du danger, du péril ou de la menace (CAstel, 2003 ; Peretti-Watel, 2000).

Ces préoccupations sont complémentaires à l'analyse économique, qui propose une définition du risque finalement similaire. L'étude du risque en économie se concentre sur l'analyse du comportement des agents face au risque (notamment en termes de préférences et d'aversion pour le risque) et sur les techniques de gestion du risque. L'évolution des mathématiques et des probabilités est indissociable de celle de l'économie du risque, et ces disciplines ont été les premières à tenter de comprendre le risque en dehors de toute connotation normative. L'étude des jeux de hasard, les techniques du calcul actuariel et les statistiques de la décision ont été mises au service de l'étude de la rationalité. Le risque désigne les événements dont on peut appréhender l'occurrence à l'aide d'une loi de probabilités. Il est entendu de manière générale comme la probabilité d'un événement combinée à l'ampleur des pertes et des gains qu'il entraîne (PrADIER, 2006). La notion d'incertitude est quant à elle privilégiée (notamment par les keynésiens) face à des événements dont la probabilité de survenance ne peut être quantifiée. Une incertitude peut donc devenir un risque dès lors que l'on dispose d'une information statistique ou probabiliste suffisante. En réalité, la frontière entre ces deux notions n'est pas nette (3) et se caractérise par un continuum de situations dont la prévision de survenue repose sur une connaissance limitée provenant de l'observation. Il ressort que la conception économique du risque est intimement liée à l'évaluation subjective qui est faite d'un événement contre lequel il devient possible et éventuellement souhaitable pour les agents de s'assurer.

Comme la notion de risque implique une approche par l'assurance, certains risques sociaux sont mieux pris en compte par la théorie économique que d'autres. On a l'archétype du risque en économie de la santé, où la maladie est un risque assurable, dont l'occurrence peut être probabilisée, et face auquel le comportement du consommateur de soin est étudié. Les risques qui ne sont jamais couverts par des mécanismes d'assurance privée, comme certains risques liés à l'emploi, la pauvreté et l'exclusion, sont moins étudiés sous cet angle. De façon générale, la littérature économique privilégie l'étude des risques auxquels font face les

(3) Sur la discussion entre incertitude et risque (en particulier chez Knight, 1921), voir Pradier (2006) et Moureau, RivaudDANSET (2004). entrepreneurs, et aborde peu les risques qui sont subis par les travailleurs (PrADIER, 2006). Cette distinction des risques en fonction des agents qui les prennent ou les subissent suffit-elle pour définir les risques dits «sociaux» d'un point de vue économique? Certes, aujourd'hui les risques sociaux touchent les consommateurs (les offreurs de travail et leurs familles), mais ils pourraient potentiellement être pris en charge exclusivement de façon privée, et il ne conviendrait plus, dans ce cas, de parler de risques «sociaux». Il existe de nombreux arguments dans la théorie économique qui peuvent justifier une prise en charge mutualisée et publique (lutte contre 1'anti-sélection, réduction des coûts, redistribution, rentabilité des investissements publics, etc.), mais des solutions privées peuvent aussi être privilégiées si les critères d'arbitrage sont différents (typiquement pour les retraites ou la maladie). Le choix d'une gestion mutualisée et publique du risque relève d'une décision politique. Dans l'approche économique aussi, la notion de risque social n'est pas objectivée à partir des caractéristiques du risque, car aucun risque n'est social en soi. Par conséquent, et pour reprendre les termes d'un juriste (DuPEYroux, 1998), les risques sociaux sont des risques économiques par nature (ils ont des conséquences économiques sur les revenus et les dépenses des agents), et ne sont «sociaux» que dans la mesure où une garantie collective est aménagée.

\section{Une tautologie nécessaire?}

La perception du risque social comme une construction sociale paraît consensuelle à travers les disciplines, et suggère une «tautologie nécessaire» pour identifier les risques sociaux. L'utilisation courante du terme de "nouveaux risques sociaux" pose alors un problème méthodologique. En effet, ils incluent certains risques qui ont récemment été intégrés par la Sécurité sociale (par exemple la dépendance qui a été reconnue comme un nouveau risque social par la législation allemande), mais aussi d'autres qui sont mal ou pas pris en charge par la protection sociale mais qui sont «appelés à l'être». Y inclure cette seconde catégorie constitue $a$ priori une acception trop extensive, voire impropre de la notion. En effet, si l'on reconnait que le risque social est le résultat d'une construction sociale, il n'est pas justifié d'appeler "sociaux» des risques qui ne le sont pas encore de facto, c'est-à-dire qui ne sont pas déterminés comme tels par le législateur. L'inclusion d'une situation parmi les nouveaux risques sociaux est alors normative, puisqu'elle implique une conception de ce qui serait souhaitable comme prise en charge par l'État Providence. Certes, l'utilisation du terme de «nouveaux risques sociaux » relève parfois du discours administratif ou politique, et on peut alors difficilement lui attribuer un manque de rigueur scientifique. Mais le flou qui entoure la notion se retrouve aussi dans des travaux 
scientifiques, qui sont dès lors plus ou moins explicitement normatifs.

Pourtant, cette tautologie heurte le sens commun qui voudrait certainement à juste titre distinguer les risques sociaux entre eux et mettre à l'écart ceux que l'on n'envisage pas comme des risques potentiellement sociaux. En effet, une conséquence de l'acception du risque comme construction sociale est de le rendre a priori potentiellement infini. Or il faut pouvoir quantifier et hiérarchiser les risques pour maîtriser une «demande infinie». Ce travail nécessiterait l'explicitation de critères d'identification, afin que les débats puissent se structurer sur des bases partagées, voire dépasser les oppositions profondes et idéologiques qui sont associées à l'utilisation de la notion de risque. Par exemple, en l'absence de critères affichés et applicables, sur quelle base refuser ou accepter de reconnaître le surendettement, les accidents de la route, la flexibilisation du marché du travail, les carrières atypiques, ou l'éviction précoce du marché du travail, ou même les divorces, comme des nouveaux risques sociaux? Sur quelle base prétendre que les nouveaux risques sociaux affectent en premier lieu les femmes, les personnes âgées, ou les jeunes, plutôt que les exclus et les immigrés?

\section{Le potentiel d'un risque à être social : essai de critériologie}

Développer une approche positive apparaît donc nécessaire avant de se placer sur le terrain normatif. C'est pourquoi nous proposons une grille dont l'ambition est d'expliciter des critères communs aux risques sociaux. Nous cherchons à délimiter le champ des risques potentiellement sociaux par des critères économiques qui nous semblent guider cette construction sociale. Comme le risque social est une construction sociale, nous reconnaissons qu'il ne peut être qualifié comme tel a priori. En effet, les conditions objectives d'une situation sociale ne suffisent pas à expliquer leur émergence comme problème social (Blumer, 1971). Cette grille ne vise donc en aucun cas à se substituer aux débats sociaux nécessaires pour la détermination effective des risques sociaux.

\section{Les dénominateurs communs des risques sociaux}

Nous nous inspirons de l'analyse d'Inge KaUL (2006) qui a montré les insuffisances de définition habituelle des biens publics et proposé une approche positive et évolutive de ce concept. En effet, si les biens publics sont caractérisés par la non-rivalité et la non-excluabilité, il n'y a en réalité pas de lien automatique entre le statut privé ou public des biens et leurs propriétés intrinsèques. Certains biens, à l'instar de la terre, peuvent tomber dans le domaine privé ou public ou encore faire la navette entre les deux. Ceci l'amène à distinguer deux niveaux de définition des biens publics : le potentiel particulier de ces biens à être publics du fait de leurs caractéristiques (non rivalité, non excluabilité, ou les deux) (1), et le caractère public de ces biens s'ils sont de facto non exclusifs, accessibles à tous les consommateurs ou les affectant tous (2).

On peut dans cette perspective assimiler les risques sociaux à des «maux sociaux», qui présentent des caractéristiques communes, mais qui ne sont pas «sociaux» en soi. On l'a dit, les risques sociaux le sont du fait de leur prise en charge collective, partiellement ou intégralement publique, à travers l'assurance sociale au sens large (4). Mais on peut aussi distinguer le potentiel des risques à être sociaux (1) du caractère véritablement social, qui se révèle dans la prise en charge et relève d'une construction sociale (2).

Nous nous intéressons ici au premier niveau de définition, qui doit permettre de délimiter un champ de risques potentiellement sociaux à partir de caractéristiques objectives communes à ces risques. Nous entendons le potentiel des risques à être sociaux comme un faisceau de caractéristiques nécessaires qui justifient une prise en charge collective. Nous en proposons quatre.

\section{Indépendance entre le risque et la responsabilité}

L'indemnisation des victimes de risques sociaux relève de régimes spéciaux qui se distinguent du droit commun de la responsabilité civile. La responsabilité civile de la personne ayant causé l'évènement n'a pas à être engagée pour que la victime soit indemnisée des conséquences du risque.

Si ce critère est sans objet pour les risques couverts par des prestations non indemnitaires (par exemple, les minima sociaux), il est néanmoins déterminant pour des risques comme les accidents du travail et les maladies professionnelles (AT/MP). En effet, l'élément fondateur du régime des AT/MP a été le principe de la responsabilité pour risque, qui permet de garantir l'indemnisation des victimes sans que la faute de l'employeur ou du salarié ne soit nécessairement recherchée. Ainsi, ces risques relèvent de la socialisation directe, dans la mesure où leur indemnisation ne passe pas par le biais de la recherche de responsabilité. Ces régimes reposent sur une certaine indépendance entre le risque et la responsabilité. Néanmoins, les deux notions ne sont pas totalement déconnectées, puisque des responsabilités peuvent être recherchées par le biais d'actions récursoires, à travers lesquelles l'assureur peut se substituer à la victime et engager la responsabilité

(4) On entend par là les mécanismes d'assurance, d'assistance, et de redistribution du système de protection sociale (cf. Blanchet, 1996) 
civile de la personne ayant commis le dommage (5) (MALAurie et al., 2009).

L'indépendance entre le risque et la responsabilité est un élément déterminant pour pouvoir qualifier un risque de «social». Ainsi, l'assurance sociale ne couvre pas les risques pour lesquels l'indemnisation nécessite une responsabilité avérée (par exemple, les victimes de produits défectueux). Il en est de même pour les risques dont l'occurrence entraîne systématiquement une recherche de responsabilité, qui peuvent néanmoins être indemnisés par un fond de garantie si le responsable est défaillant (par exemple les accidents de la circulation). Ce critère n'est toutefois pas suffisant : certains risques tels que le terrorisme, les catastrophes naturelles ou le SIDA peuvent être socialisés et offrir une garantie aux victimes, y compris en l'absence de responsables potentiels.

\section{Les conséquences économiques de la réalisation du risque}

Cet élément de définition permet d'insister sur le fait que le dénominateur commun des risques sociaux n'est pas à trouver dans leurs causes mais dans leurs conséquences. DupeYroux (1998) rappelle à juste titre qu'il est erroné de considérer comme nécessairement sociaux les «risques inhérents à la vie sociale». En revanche, les risques sociaux ont tous des effets similaires : ils affectent la sécurité économique des individus, en limitant leurs capacités de gains ou en engendrant un accroissement exceptionnel de leurs dépenses. Dans cette perspective, la définition de nouveaux risques sociaux résultant des transformations de la société post-industrielle ( $c f$. supra) est insatisfaisante car elle met l'accent sur les causes de ces nouvelles situations. On peut admettre que les causes ont changé, que la lutte contre celles-ci est prioritaire, et qu'elles sont déterminantes pour influencer les choix politiques. Mais les causes sont secondaires pour définir les risques sociaux, car elles n'en sont pas une caractéristique essentielle.

Les risques sociaux sont par nature des risques économiques, qui affectent les ressources ou les dépenses de personnes physiques (les ménages composés des travailleurs et de leurs familles, ou les citoyens). Les risques dont les conséquences sur les individus sont indirectes, car ils affectent en premier lieu les biens (par exemple le risque habitation) ou les personnes morales (par exemple la crise économique), relèvent a priori d'un autre champ. Du point de vue des conséquences matérielles, on peut distinguer deux catégories de risques sociaux : les risques «physiques» qui réduisent la capacité de gain (chômage, invalidité, vieillesse, etc.) et les

(5) Par exemple en direction de l'employeur potentiellement responsable de l'accident du travail. risques « économiques » qui accroissent les dépenses (par exemple le logement, la maladie) (DupeYroux, 1998). Ces conséquences économiques doivent pouvoir être exprimées en unités monétaires et pouvoir être compensées en nature ou en espèces. De plus, elles peuvent être immédiates ou différées dans le temps par rapport à l'événement déclencheur (par exemple les droits à la retraite dépendent de l'ensemble de la carrière). Nous rappelons que la protection sociale a pour objectif de réparer les risques, et que la prévention relève d'autres politiques sociales : la compensation se fait donc $a$ posteriori.

\section{Le caractère assurable du risque}

Le risque est un événement aléatoire (6) assurable à partir du moment où l'on dispose de l'information et des techniques nécessaires, et en premier lieu de la faculté d'en calculer la probabilité d'occurrence et d'en estimer le coût de couverture.

De plus, une des spécificités de l'assurance sociale est de prendre en charge les risques qui sont susceptibles de toucher une fraction importante ou/ et indéterminée de la population. C'est à la fois une condition de légitimité politique et d'efficacité. En effet, comme le note Didier Blanchet (1996), la spécificité de la couverture sociale est notamment légitimée par le soutien social qu'elle reçoit et qui est nécessaire à sa pérennité, et par le besoin de proposer une couverture aussi extensive que possible. Ainsi, la couverture sociale peut être préférée à une couverture privée pour les risques dont la probabilité d'occurrence est potentiellement élevée, mais qui sont difficilement délimitables. Le risque de vieillesse et de décès en est un exemple : réparties sur l'ensemble de la population, les assurances privées peuvent se développer facilement lorsque le risque est aisément délimitable (par exemple l'assurance-vie), mais plus difficilement lorsque sa nature est difficile à établir et que ses tendances à long terme sont difficilement évaluables (par exemple, la dépendance) (BLANCHET, 1996).

Toutefois, tout système d'assurance peut être mis en échec par des risques dont la probabilité d'occurrence est inconnue ou dont le coût de prise en charge est potentiellement exorbitant. En particulier, les risques de catastrophe, rares, fortement corrélés et donc potentiellement très coûteux (par exemple les pandémies), ont moins intérêt à être pris en charge par un système assurantiel (qui doit répercuter ce coût sur les primes ou les cotisations des assurés) que directement par 1'État (par exemple à travers des impôts non affectés aux dépenses).

(6) En matière de risques sociaux, l'occurrence est parfois quasi certaine (par exemple la maladie ou la vieillesse) et l'aléa se situe alors davantage dans la date de survenue, la fréquence, ou la durée de l'événement. 
Enfin, une maîtrise suffisante de l'aléa moral est nécessaire. En effet, tout mécanisme d'assurance ou de transfert peut conduire à une modification des comportements des agents qui, se sachant couverts, peuvent accroître la probabilité d'occurrence du risque (risque moral ex ante), ou le coût de la prise en charge après la survenance du risque (risque moral ex post). On peut noter que l'ampleur de cet effet théorique, qui pose l'hypothèse du contrôle de leur situation par les individus, reste difficile à mesurer empiriquement(7). Ceci invite donc à une certaine prudence, puisque l'aléa moral peut théoriquement rendre la responsabilité sociale inefficace, et ainsi permettre de justifier la réduction de la couverture de nombreux risques comme la pauvreté, pour lesquels de tels effets ne seraient pourtant pas avérés empiriquement (BAKER, 1996). L'assurance sociale dispose globalement des mêmes outils que l'assurance privée pour maîtriser l'aléa moral. Ses principaux mécanismes en sont la modulation de l'indemnisation par le contrôle de la responsabilité (par exemple, la cause du chômage) et les incitations ou pénalités financières (intéressement, franchises, ticket modérateur, bonus-malus, etc.). Lorsque l'aléa moral est très élevé et que les systèmes d'assurance ne disposent pas d'outils efficaces pour le maitriser (par exemple pour le surendettement), l'objectif d'efficacité conduit à privilégier la responsabilisation individuelle à la mutualisation.

Il faut noter que le système de protection sociale n'a pas pour objectif d'empêcher la prise de risque des individus. Il peut au contraire l'autoriser, voire la favoriser, dans la mesure où les modifications de comportements ne sont porteuses d'effets pervers qu'au-delà d'une certaine limite (BLANCHET, 1996), et qu'un accroissement de la consommation produit aussi des externalités positives (BAKER, 1996). Il s'agit donc de trouver un équilibre entre des garanties permettant aux individus de prendre certains risques et la maîtrise de comportements opportunistes. Ainsi, une durée d'indemnisation du chômage relativement longue peut favoriser un meilleur appariement sur le marché du travail, de même qu'une indemnisation relativement généreuse peut permettre de soutenir l'exercice de professions très exposées aux interruptions de travail (par exemple, les intermittents du spectacle). Même une responsabilité individuelle contrôlée et avérée n'empêche pas la couverture du risque : les salariés démissionnaires allemands sont par exemple indemnisés au titre du chômage après une suspension de l'indemnisation qui ne dépasse pas trois mois. De même, certains événements (accidents, maladies) consécutifs à des conduites à risques (sports extrêmes,

(7) L'expérience de la RAND Corporation en 1974 reste à ce titre exceptionnelle puisque cette expérimentation avec tirage aléatoire de la couverture a permis de mesurer précisément l'élasticité du prix de la demande de soins et donc l'effet incitatif du co-paiement sur la consommation (GEOFFARD, 2006). consommation de drogues) peuvent être couverts au même titre que d'autres événements de santé.

\section{Une répartition socialement inégale}

Les individus ne sont pas égaux devant les risques sociaux, même s'ils sont tous susceptibles d'y être exposés. En effet, les risques ne sont pas répartis de façon aléatoire entre les individus et les groupes sociaux. Ainsi, les inégalités d'exposition au risque se cumulent avec des inégalités de revenu : par exemple, les risques de chômage ou d'hospitalisation sont plus élevés pour les ouvriers et les employés que pour les cadres. Par conséquent, si la tarification était actuariellement neutre, certaines catégories de population à haut niveau de risque et/ ou à bas niveau de revenu, ne pourraient pas s'assurer individuellement, le coût de la prime d'assurance étant trop élevé. En d'autres termes, la neutralité actuarielle, caractéristique de l'assurance privée, conduit à exclure de la couverture certaines catégories de la population. Ces inégalités de risque et de revenu justifient une prise en charge par l'assurance (ou l'assistance) sociale. Par exemple, des dispositifs spécifiques de couverture maladie permettent la couverture des hauts niveaux de risques (Medicare pour les personnes âgées aux États-Unis) ou des bas niveaux de revenus (Medicaid américain, CMU et AME en France). Contrairement à l'assurance privée, l'assurance sociale crée toujours de la redistribution par un partage du risque entre les classes de risques (redistribution horizontale) et entre les classes de revenu (redistribution verticale). Ainsi, même lorsque son mécanisme est proche de la neutralité actuarielle (par exemple les retraites en compte notionnel en Suède dont le fonctionnement est celui d'une capitalisation fictive), elle contient toujours des mécanismes de compensation qui créent de la redistribution (par exemple la validation des périodes de chômage, de maladie ou de congés maternités).

L'objectif de redistribution de l'assurance sociale va de pair avec une obligation d'assurance qui permet de lutter efficacement contre les phénomènes d'anti-sélection auxquels tout système d'assurance est confronté du fait de l'asymétrie d'information. Comme les individus sont a priori mieux renseignés sur leur niveau de risque que l'assureur, à une prime d'assurance donnée, les «mauvais risques» (les individus dont le niveau de risque multiplié par le coût de couverture du risque est supérieur à la prime) auront intérêt à s'assurer, alors que les «bons risques» préfèreront l'auto-assurance. Or, la viabilité financière d'un système de prise en charge offrant des garanties de qualité avec des primes abordables nécessite l'affiliation des bons risques. La conciliation de ces deux objectifs justifie une régulation des assurances par l'intervention publique. Les mécanismes de solidarité (par exemple la double obligation d'assurance, la tarification en fonction 
des capacités contributives) qu'elle peut imposer sont autant de moyens simples et efficaces de lutter contre les effets de l'anti-sélection sur l'équité, l'efficacité, et la qualité de couverture(8).

Contrairement aux biens publics, traditionnellement définis à partir de leurs propriétés fondamentales, les risques sociaux le sont traditionnellement a posteriori, du fait de leur prise en charge. Ces quatre critères nous semblent permettre de délimiter a priori le champ des risques potentiellement sociaux. C'est le second niveau de la définition, à savoir la prise en charge, qui détermine réellement l'aspect social des risques, et qui ne peut être défini qu'a posteriori. De la même façon que les biens publics, qui sont les biens dont la consommation est de facto accessible à tous, les risques sociaux ont vocation à être pris en charge de façon universelle. Le rôle de l'État est de s'assurer de cette finalité, mais les mécanismes peuvent être publics ou privés. En effet, les risques qui entrent dans le domaine de la protection sociale ne sont pas pour autant systématiquement couverts par l'État, et les frontières sont complexes entre prise en charge mutualisée privée et collective, qui peuvent se substituer l'une à l'autre ou coexister. La prise en charge des risques sociaux peut prendre des formes très diverses mais elle est au moins partiellement publique, garantie par l'État, et elle offre une couverture généralisée, institutionnalisée et systématisée selon des critères définis.

\section{Le caractère social du risque : un terrain normatif à expliciter}

Cette approche peut servir à déterminer, parmi des «nouveaux risques», lesquels sont potentiellement «sociaux», en admettant qu'ils ne pourront réellement être qualifiés comme tels que lorsque le critère de la prise en charge aura été rempli et qu'une responsabilité collective aura été reconnue. La notion de responsabilité est centrale, et, en fonction de ce que l'on reconnaît comme étant du ressort de la collectivité, le risque peut devenir ou non un risque social. Cependant, on peut aussi envisager le processus inverse. L'idée de l'amélioration de l'information des individus, qui leur permet d'avoir une meilleure conscience des risques, peut aussi conduire à les rendre progressivement plus responsables. Les situations néfastes qui les atteignent ne sont alors plus le fait de processus sociaux plus larges, mais sont induites par des choix rationnels manqués. On peut dès lors imaginer que des risques sociaux aujourd'hui puissent ne plus l'être demain, car la responsabilité de ces risques aura été attribuée aux individus.

(8) Pour une démonstration concernant l'assurance santé, cf. Marchand, Pestieau (1996).
Chacun des critères d'identification proposés ci-dessus peut appeler à des réponses différentes en fonction des seuils qui sont considérés comme acceptables ou souhaitables.

À partir de quelle ampleur les conséquences matérielles sont-elles considérées comme suffisamment importantes pour être prises en charge socialement? Les pertes de bien-être ne justifient pas une intervention collective si elles sont considérées comme inévitables dans un environnement risqué et si elles incitent à adopter des comportements vertueux. De même, quelles sont les inégalités qui sont acceptées par la société? Quelles sont celles contre lesquelles elle décide de lutter? La redistribution ne sera justifiée que si les pertes des uns sont compensées par les gains des autres. La question de la prise en charge ou non par la collectivité est une question normative qui appelle une réponse variable, suivant le niveau de richesse des États et de l'idéologie dominante en matière de justice et d'équité.

Le constat et les listes de nouveaux risques sociaux sont souvent dressés sans que ne soit explicité à quel titre on appelle à la prise en charge de certains risques plutôt que d'autres. L'approche positive des risques sociaux ne se limite pas à l'identification des risques et doit s'accompagner de l'explicitation des critères de quantification et de hiérarchisation des risques. En effet, selon que l'on utilise les critères de quantification de la nouvelle économie du bien-être et que l'on mesure les pertes de bien-être en niveaux de vie, ou ceux de l'économie du bonheur qui propose de les mettre en perspective avec les pertes de bien-être en termes de satisfaction, on peut aboutir à des résultats et des recommandations politiques contrastées (DAVOINE, 2009). De même, selon le critère d'appréciation de l'optimalité d'une répartition, les recommandations de redistribution diffèrent : une situation optimale au sens de Pareto (dans laquelle le bien-être d'un individu ne peut être augmenté sans détériorer celui d'un autre), qui limite les possibilités de redistribution, peut néanmoins inviter à une redistribution selon le critère de différence de John RAWLS (où une mesure de correction des inégalités est juste si elle améliore le bien-être du groupe le moins avantagé), critère à son tour insuffisant au sens de Amartya SEN ou de Ronald Dworkin, pour lesquels il convient de maximiser l'égalité réelle (DAvorne, 2009, Schmid, 2006).

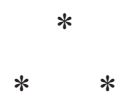

Cet article s'est attaché à montrer qu'une approche positive des nouveaux risques sociaux est à la fois nécessaire - car l'acception courante des nouveaux risques sociaux est paradoxale et ravive les débats idéologiques autour de la réforme de la protection sociale -, et possible, puisque, tout en reconnaissant que le risque social est le 
fruit d'une construction sociale, certaines caractéristiques objectives permettent de délimiter le champ des risques potentiellement sociaux. Dans quelle mesure les éléments d'identification proposés permettent-ils de mettre en exergue les habits neufs des risques sociaux? Le critère d'indépendance entre le risque et la responsabilité écarte du champ des risques tels que les accidents de la route, pour lesquels la responsabilité individuelle est systématiquement recherchée. Le critère des conséquences économiques écarte du champ les situations sur le marché du travail (risques d'inactivité ou temps partiel subis, de carrières atypiques, d'instabilité de l'emploi, de flexibilité, d'obsolescence des compétences) dont les conséquences sur le bien-être matériel sont indirectes. Ainsi, la sécurisation des parcours relève a priori du champ de la politique de l'emploi. En revanche, ces situations peuvent être couvertes par la protection sociale au titre des risques conduisant directement à une perte de revenus. Plus précisément, ce sont les risques de chômage non indemnisé et/ou de chômage de longue durée, de pauvreté et d'exclusion auxquels les travailleurs précaires sont exposés, comme peuvent l'être des populations n'ayant pas accès à certains droits (jeunes, migrants), qui peuvent quant à eux être des candidats à une extension de la couverture par la protection sociale. De même, la privatisation des retraites n'est pas en soi un risque potentiellement social, alors qu'un risque de faibles niveaux de retraites ou d'absence de droits à la retraite peut l'être. Les risques qui affectent des personnes morales ou des biens ne semblent quant à eux pas être candidats à une couverture sociale. Le caractère probabilisable et la maîtrise de l'aléa moral conduisent à exclure a priori, d'une part les risques de catastrophe, d'autre part les risques pour lesquels la responsabilité individuelle est élevée et la prise de risque peu porteuse d'externalités positives (à l'instar du surendettement). Enfin, le critère de la répartition socialement inégale constitue également un argument pour exclure de la couverture sociale certains risques liés à l'instabilité de la structure familiale (par exemple les frais de divorce), mais pour y inclure d'autres risques qui en découlent comme la monoparentalité, ainsi que les risques de faiblesse des ressources ou d'absence de droits de certains groupes (par exemple les migrants, jeunes ou inactifs). 


\section{Bibliographie}

BAKER T. (1996), "On the Genealogy of Moral Hazard”, Texas Law Review, VOL. 75, NO 2, pp. 237-292.

BECK U. (1986), Risikogesellschaft, Frankfurt a/M, Suhrkamp Verlag. (traduction française en 2001 sous le titre La société du risque: Sur la voie d'une autre modernité).

BlAnChET D. (1996), «La référence assurantielle en matière de protection sociale : Apports et limites», Économie et Statistique, no 291-292, pp. 33-45.

Blumer H. (1971), "Social Problems as Collective Behavior”, Social Problems, vol. 18, no. 3, pp. 298-306.

Bonoli G. (2006), "New Social Risks and the Politics of Postindustrial Social Policies", The Politics of Postindustrial Welfare State: Adapting post-war policies to new social risks, Routledge.

CASTEL R. (2003), L'insécurité sociale : Qu'est-ce qu'être protégé? La République des idées, Seuil.

Concialdi P. (1999), «Pour une économie politique de la protection sociale», Revue de l'IRES, no 30, vol. 2, pp. 1-39.

Davorne L. (2009), «L'économie du bonheur: Quel intérêt pour les politiques publiques?», Revue Économique, vol. 60, no. 4, pp. 905-926.

Dupeyroux J-J. (1998), Droit de la sécurité sociale, Paris, Dalloz (13e éd.).

Ewald F. (1986), L’État Providence, Grasset.

Esping-Andersen G., Gallie D., Hemerijck A., Myles J. (2001), A New Welfare Architecture for Europe? Rapport à la présidence belge de l'Union européenne.

Ferrera M., Rhodes M. (2000), "Building a Sustainable Welfare State: Reconciling Social Justice and Growth in the Advanced Economies", Recasting European Welfare States, London, Frank Cass.

Foucault M. (1977), Sécurité, Territoire, Population, Cours au Collège de France.

Geoffard P-Y. (2006), La lancinante réforme de l'assurance maladie, coll. CEPREMAP, Presses de l'ENS.

Giddens A. (1998), The Third Way: The Renewal of Social Democracy, Polity Press \& Blackwell publishers.

Holzmann R., Jorgensen S. (2001), "Social Risk Management: A New Conceptual Framework for Social
Protection, and Beyond", International Tax and Public Finance, no 8, pp. 529-556.

KaUl I. (2006), «Une analyse positive des biens publics », L'avancée des biens publics, pp. 23-54, Paris, Bibliothèque Albin Michel Économie.

Kessler D. (1999), "Social Security and Private Insurance: The Great Change", The Geneva Papers on Risk and Insurance, vol. 24, no 4, pp. 439-447.

Kessler F. (2000), Droit de la protection sociale, Dalloz, Paris.

Knight F. (1921), Risk, Uncertainty and Profit, Hart, Schaffner \& Marx; Boston: Houghton Mifflin Company, The Riverside Press, Cambridge.

Malaurie P., Aynès L., Stoffel-Munck P. (2009), Les obligations (4e éd.), coll. Droit civil, Paris : Defrénois, Lextenso.

Marchand M., Pestieau P., (1996), «L'État ou le marché dans l'assurance maladie», Revue Française d'Économie, vol. 11, no 1, pp. 3-19.

Moureau N., Rivaud-Danset D. (2004), L'incertitude dans les théories économiques, Repères, La découverte, Paris.

Palier B. (2002), Gouverner la Sécurité Sociale, PUF.

Peretti-Watel P. (2000), Sociologie du risque, Armand Colin.

Pradier P.-C. (2006), La notion de risque en économie, Repères, La découverte, Paris.

RAmaux C. (2007), «Quelle théorie pour l’État Social? Apports et limites de la référence assurantielle», Revue française des affaires sociales, no 1, pp. 13-34.

Rosanvallon P. (1995), La nouvelle question sociale : Repenser l'État Providence, Paris, Seuil.

Schmid G. (2006), "Social Risk Management through Transitional Labour Markets", Socio-Economic Review, vol. 1, pp. 1-33.

Taylor-Gooby P. (ed.) (2004), New Risks, New Welfare: The Transformation of the European Welfare State, Oxford University Press.

Taylor-Gooby P., Zinn J. (2006), Risk in Social Science, Oxford University Press. 